\title{
Ischemic Optic Neuropathy Following Cardiopulmonary Bypass Surgery: A Case Report
}

\author{
Nursen Oncel Acira, c, Ahmet Kuzgun ${ }^{\mathrm{b}}$, Fatih Aygun ${ }^{\mathrm{b}}$, Zeynep Dadaci ${ }^{\mathrm{a}}$, Mehmet Borazan ${ }^{\mathrm{a}}$
}

\begin{abstract}
Postoperative visual dysfunction following major surgeries is still a significant clinical problem. Especially, ischemic optic neuropathy (ION) after cardiac surgery is a devastating complication that is known as a source of permanent visual loss. In this case study, a patient who presented with features of bilateral anterior ION after cardiopulmonary bypass surgery (CPBS) was reported.
\end{abstract}

Keywords: Ischemic optic neuropathy; Cardiopulmonary bypass; Postoperative visual dysfunction

\section{Introduction}

Postoperarative visual loss after surgery unrelated to the orbit is a rare but devastating complication following major surgical procedures. The highest rate of postoperative visual loss is reported after cardiac and spine surgeries. The major cause of postoperative visual loss is ischemic optic neuropathy (ION). Here we are reporting a case of ION bilaterally following cardiopulmonary bypass surgery.

\section{Case Report}

A 57-year-old male patient referred to Department of Ophthalmology from the Department of Cardiovascular Surgery with the complaint of blurred vision in his both eyes continuing for 1 day. He had a history of conventional cardiopulmonary by-

\footnotetext{
Manuscript accepted for publication December 03, 2014

aDepartment of Ophthalmology, School of Medicine, Mevlana University, Konya, Turkey

bDepartment of Cardiovascular Surgery, School of Medicine, Mevlana University, Konya, Turkey

${ }^{\mathrm{c} C}$ Corresponding Author: Nursen Oncel Acir, Department of Ophthalmology, School of Medicine, Mevlana University, Gemalmaz Mh. Dokumcu Sk. No. 7 Meram, Konya, Turkey. Email: nursen74@yahoo.com
}

doi: http://dx.doi.org/10.14740/jmc2016w pass surgery (CPBS) which was performed 3 days previously as a result of multi-vessel coronary artery disease. The patient had no history of drug consumption and ocular or systemic disease, but he was a heavy smoker and had positive family history of coronary artery disease. All blood tests were within normal range, preoperatively. According to preoperative records, under rutine anesthesia, the surgical procedure in which fivevessel total cardiopulmonary bypass was conducted successfully in estimated time without complication. The postoperative period in the cardiac surgical intensive care unit (CSICU) was uneventful, hematologic parameters were as expected as normal and routine treatment and follow-up procedures had been administered after surgery.

On the third day following surgery, the ophthalmologic review was organized on condition that the patient suffered poor vision as mentioned above. The examination revealed visual acuity was $2 / 20$ in both eyes. Anterior segment examination revealed no pathologic changes. The fundus examination of both eyes showed bilateral disc pallor, and indistinct disc margins (Fig. 1). Fundus fluorescein angiography revealed normal reti-

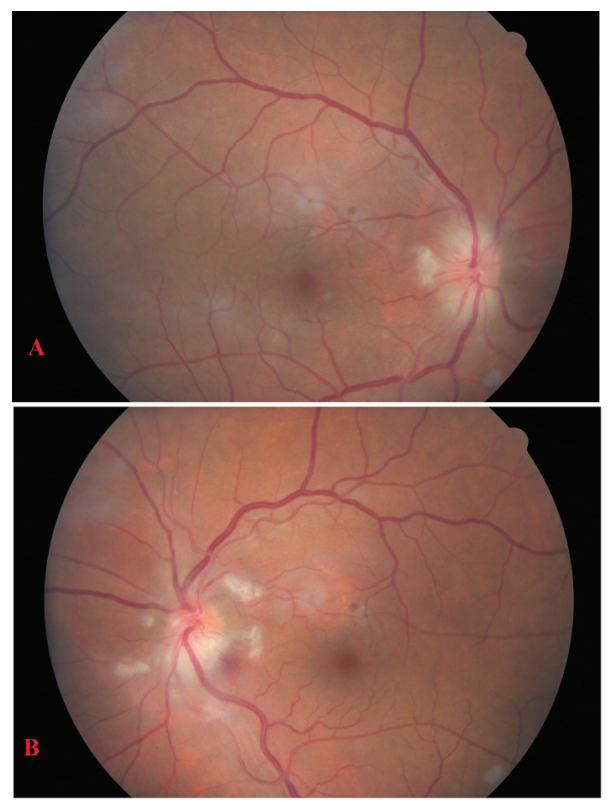

Figure 1. Bilateral optic disc swelling at presentation (A: right eye; B: left eye). 


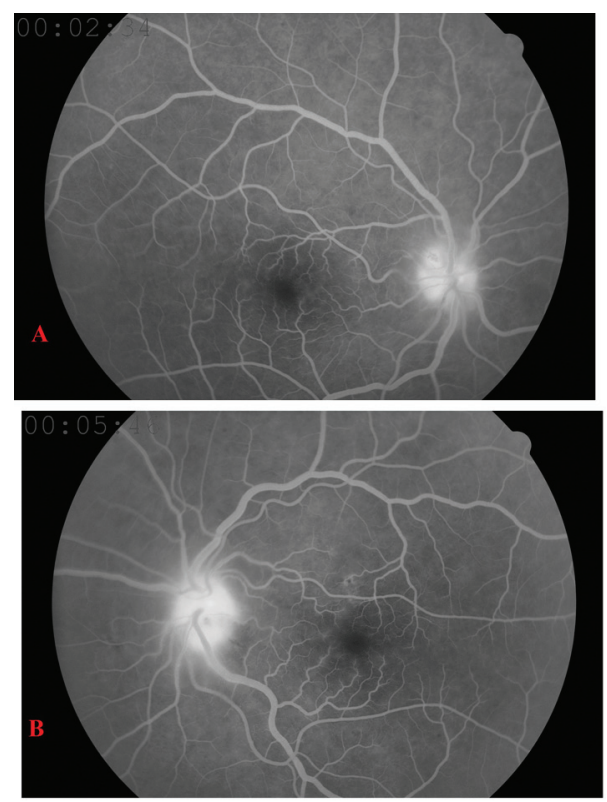

Figure 2. Late phase fluorescein angiogram of both eyes shows leakage of dye (A: right eye B: left eye).

nal appearance, however, a marked leakage on the optic nerve of the both eyes (Fig. 2). Computerized perimeter showed constricted visual field in both eyes (Fig. 3). The patient was diagnosed as ION and pulse dose steroid $(1,000 \mathrm{mg} /$ day in four equal doses) and flaxiparine $(0.4 \mathrm{mg} /$ day $)$ were administered in addition to postoperative therapy in CSICU. Three days later, the patient's visual acuity was $8 / 10$ in both eyes and the steroid dose was converted $80 \mathrm{mg} /$ day. On the 10th day, the patient's visual acuity remained stable with arcuate visual field defect in both eyes (Fig. 4) and he was discharged from the hospital with steroid $(10 \mathrm{mg} /$ day $)$ and acetylsalicylic acid $(100 \mathrm{mg} /$ day $)$ in addition to his postsurgical therapy. Weekly ophthalmological examination continued for 1 month. Then, steroid was stopped, but the patient was asked to continue oral acetylsalicylic acid and follow-up visits.

\section{Discussion}

Postoperative visual dysfunction is a well-recognized complication of cardiac surgery and usually appears in three types: infarction in occipital cortex, retinal emboli/ischemia, and ION $[1,2]$. ION is a clinical entity which can be a cause of blindness or seriously impaired vision and consists primarily of two types: anterior and posterior. These terms represent the site and ischemic nature of the lesion in the optic nerve [3]. The anterior portion of the optic nerve is supplied by the short posterior ciliary arteries, end arteries of ophthalmic artery; however, the posterior part of is nourished via the pial plexus. Compared with the anterior portion, the posterior optic nerve is more susceptible to decreased oxygen delivery and ischemia. Although anterior and posterior ION comprises different etiologies, pathogenesis, clinical features and managements, both can be detected as a complication of major surgeries, namely postoperative ION [4].

Postoperative ION is not only associated with cardiac surgeries especially with CPBS, but also could occur following some major surgeries particularly direct orbital compression in spinal procedures with the patient in the prone position and other orthopedic procedures, radical neck dissections, and thoracotomy for hemothorax [4]. Nuttall et al [5] reported incidence of ION in patients undergoing CPBS is from $0.06 \%$ to $1.3 \%$. There are several reasons that may cause ION following CPBS. The main factor is severe arterial hypotension which can occur due to prolonged general anesthesia, and massive blood loss [4]. Thromboembolism is another cause which can result from manipulation of the aorta, carbon dioxide management, and type of oxygenator used in the procedure. In addition, postoperative hemoglobin levels because of hemodilution by the pump prime, or from administration of a large amount of intravenous fluids to compensate for the blood loss may consequently lead to reduced oxygen supply and lead to ischemia in optic nerve. The other factors may predispose the optic nerve to ischemia including hypothermia which is commonly used in CPBS and resultant decrease in blood flow, and activation of complement system which resulted from extracorporeal circulation of blood flow within prosthetic membranes during CPBS. All of those conditions can consequently lead to disturbance of coagulation and homeostasis [6].

The clinical features of our patient in this report are suggestive of anterior ION. Anterior ION typically presents with diminished vision, field defects and swollen optic disc; however, posterior ION presents with a vision loss despite of initially normal disc and fundus appearance. Besides, it was also confirmed with significantly increased visual acuity that responded the therapy because posterior ION usually tends to cause bilateral massive visual loss which is permanent and no treatment has been found to be effective to recover [4].

We believe this patient had ION precipitated by his hemodynamic changes and hemodilution related surgery and we treated our patient with pulse dose steroid because he had severe disc edema which may threat visual prognosis. One can suggest that treating the underlying anemia and correcting hypotension may be sufficient therapy or visual function may be recovered spontaneously. The role of steroid therapy in ION is controversial. In a large study in patients with ION, it has been shown that while the visual improvement is $70 \%$ in treated with steroid group, it is $41 \%$ in the untreated group. Similarly, a comparison of treated versus untreated group also showed that optic disc edema resolved significantly faster in the treated group [4]. We administered high dose steroid to patient in CSICU with careful monitoring of symptoms and possible side effects of steroids. So, we propose that the improvement in vision was induced by the steroids; besides, other modalities may aid in recovery.

In conclusion, ION may occur as a complication following routine, seemingly uncomplicated cardiac surgeries, but it may cause severe visual loss. Thus, every effort should be made to correct hemodynamic parameters besides proper treatment in patients in whom ION occurs in postsurgical period. So, anesthesiologists and surgeons should be aware of this complica- 


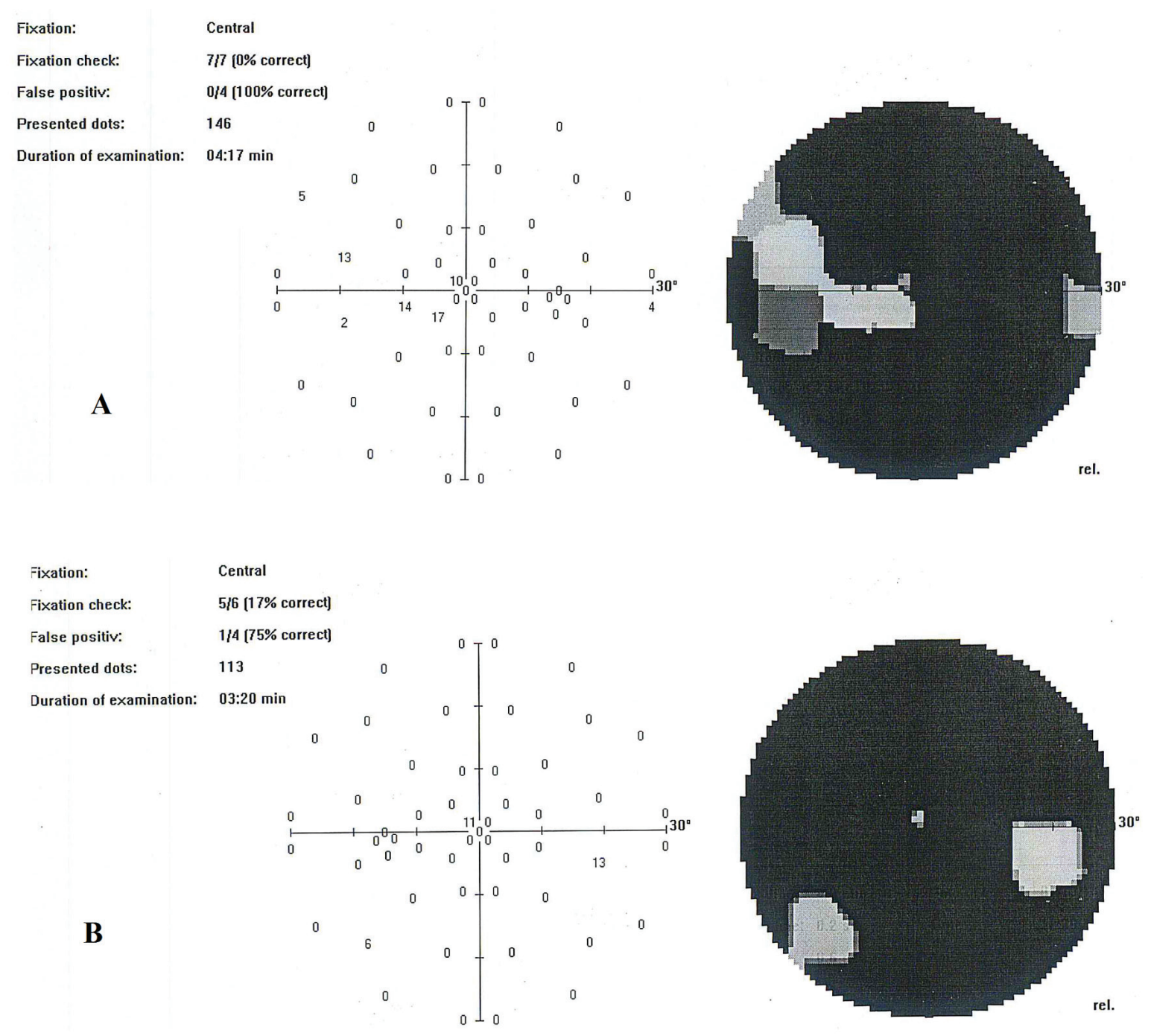

Figure 3. Automated perimetry shows constricted visual field in both eyes, leaving a small island of vision (A: right eye; B: left eye).

tion and should work in collaboration with ophthalmologists to manage it.

\section{Financial Disclosure}

No financial support was received for this submission.

\section{References}

1. Larkin DF, Wood AE, Neligan M, Eustace P. Ischaemic optic neuropathy complicating cardiopulmonary bypass. Br J Ophthalmol. 1987;71(5):344-347.

2. Hayashi H, Kawaguchi M, Okamoto M, Hasuwa K, Matsuura T, Taniguchi S, Furuya H. Asymptomatic and symptomatic postoperative visual dysfunction after cardiovascular surgery with cardiopulmonary bypass: a smallsized prospective observational study. J Cardiothorac Vasc Anesth. 2013;27(5):884-889.

3. Nenekidis I, Pournaras CJ, Tsironi E, Tsilimingas N. Vision impairment during cardiac surgery and extracorporeal circulation: current understanding and the need for further investigation. Acta Ophthalmol. 2012;90(3):e168172.

4. Hayreh SS. Management of ischemic optic neuropathies. Indian J Ophthalmol. 2011;59(2):123-136.

5. Nuttall GA, Garrity JA, Dearani JA, Abel MD, Schroeder DR, Mullany CJ. Risk factors for ischemic optic neuropathy after cardiopulmonary bypass: a matched case/control study. Anesth Analg. 2001;93(6):1410-1416, table of contents. 


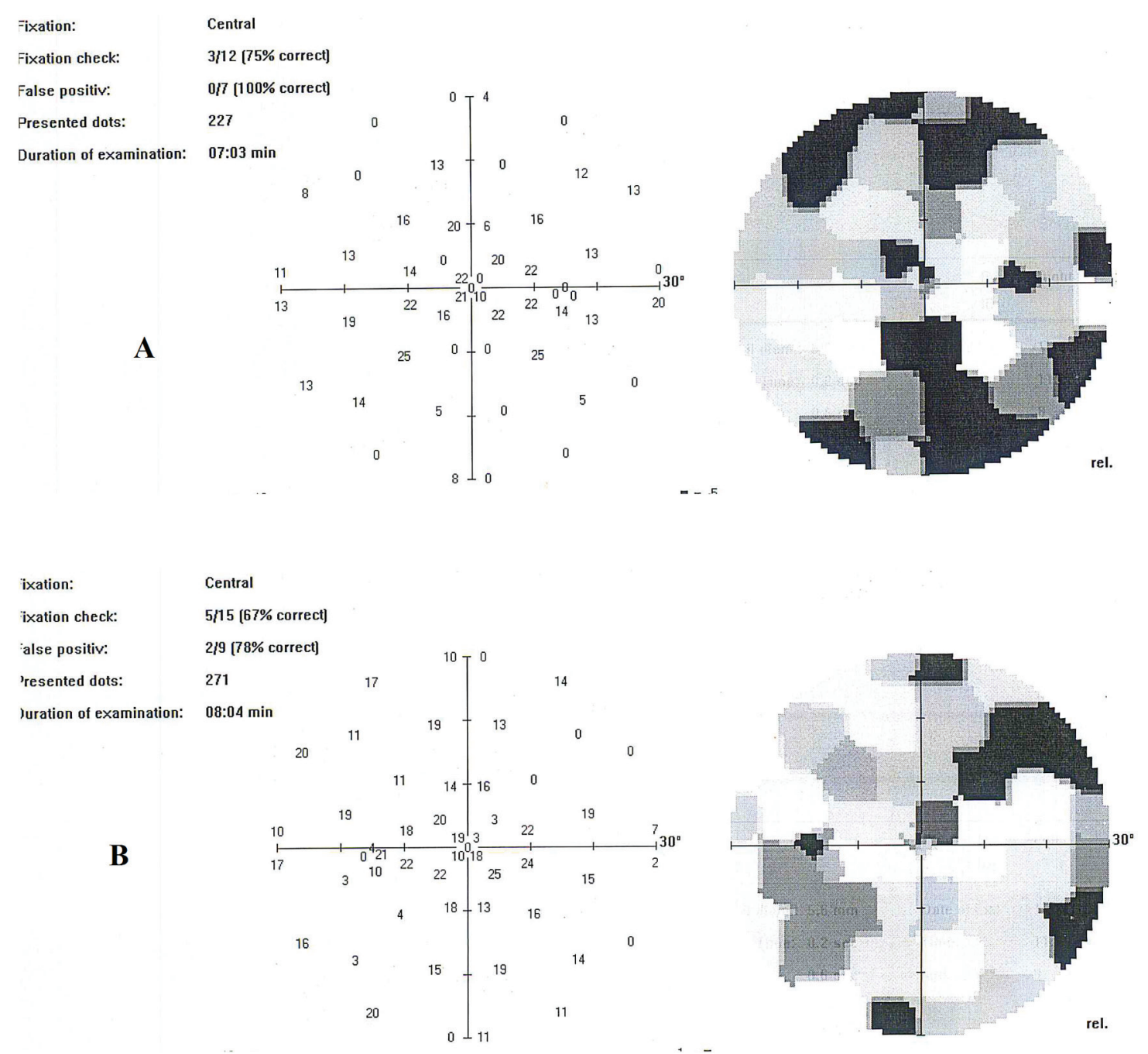

Figure 4. Arcuate scotoma defect in visual field with visual recovery in both eyes (A: right eye; B: left eye).

6. Kalyani SD, Miller NR, Dong LM, Baumgartner WA, Alejo DE, Gilbert TB. Incidence of and risk factors for perioperative optic neuropathy after cardiac surgery. Ann Thorac Surg. 2004;78(1):34-37. 